\title{
Role of Scheimpflug Imaging in the Diagnosis of Intraocular Lens Damage after Foldable Lens Implant: Case Report
}

\author{
Txomin Alberdi ${ }^{1}$, Javier Mendicute ${ }^{1}$, Lucía Bascarán ${ }^{1}$, Juaristi $L^{1}$ and Javier Ruiz-Ederra ${ }^{2}$ \\ ${ }^{1}$ Ophthalmology Department, Donostia University Hospital, Donostia-San Sebastian, Spain \\ ${ }^{2}$ Biodonostia Health Research Institute, Donostia-San Sebastian, Spain
}

*Corresponding author: Prof. Txomin Alberdi, Ophthalmology Department, Donostia University Hospital, Donostia-San Sebastian, Paseo Dr. Beguiristain s/n, Donostia-San Sebastian, 20014, Spain, Tel: +34665729017, Fax: 943007452, E-mail: txotere@gmail.com

\begin{abstract}
To our knowledge, this is the only article that presents the Peak densitometry method of the Pentacam HR software as a tool for the diagnosis of intraocular lens damage after cataract surgery.
\end{abstract}

\section{Keywords}

Scheimpflug Imaging, Intraocular lens damage, Peak densitometry method

\section{Introduction}

In 2004, Hayashi, et al. first obtained a Scheimpflug image of the anterior segment at the $0^{\circ}, 45^{\circ}, 90^{\circ}$ and 135-degree meridians for posterior capsule opacification measuring. One decade later, the new Pentacam HR software provides a 3-Dimensional segment analysis (angle, depth and volume), and its densitometry software that includes nucleus staging software and 3 densitometry methods (area, linear, and peak) to measure density of any anterior chamber structures (cornea, lens, anterior capsule, posterior capsule...), and provides objective quantitative continuous values in an easy, rapid, and repeatable manner [1,2]. 25 Scheimpflug images are acquired during each scan. A second front pupillary camera aligns the images and compensates for eye movement. The measurement process takes less than 2 seconds, and small eye-movements are captured and corrected simultaneously. The Scheimpflug device calculates a quality specification score that takes into account the area covered, alignment and eye movement.
This score helps users evaluate the validity of each test data [1].

Area and Linear densitometry methods are increasingly used methods to measure density of any structure of the anterior segment. The peak densitometry value is the point at which the lens densitometry is highest on the densitogram. Therefore, it is not useful to measure the average density of structures of the anterior segment; however, it has other utilities like the one we describe in this article.

The trend in cataract surgery has been to insert lenses through smaller incisions for faster recovery and to generate smaller magnitudes of astigmatism. This has been possible because the lenses have been folded or rolled up and then injected. Because of this increased manipulation, intraocular lenses are more prone to damage [3].

The aim of this article is to propose the density levels provided by Oculus Pentacam ${ }^{\circ} \mathrm{HR}$ densitometry Peak method as a diagnostic tool to evaluate lens damage after cataract surgery.

\section{Case Report}

A 70-year-old woman referred positive dysphotopsias after cataract procedure. Ophthalmologic examination reveals a $20 / 20$ visual acuity and showed a thin marked line on the intraocular lens and no other find of interest. 


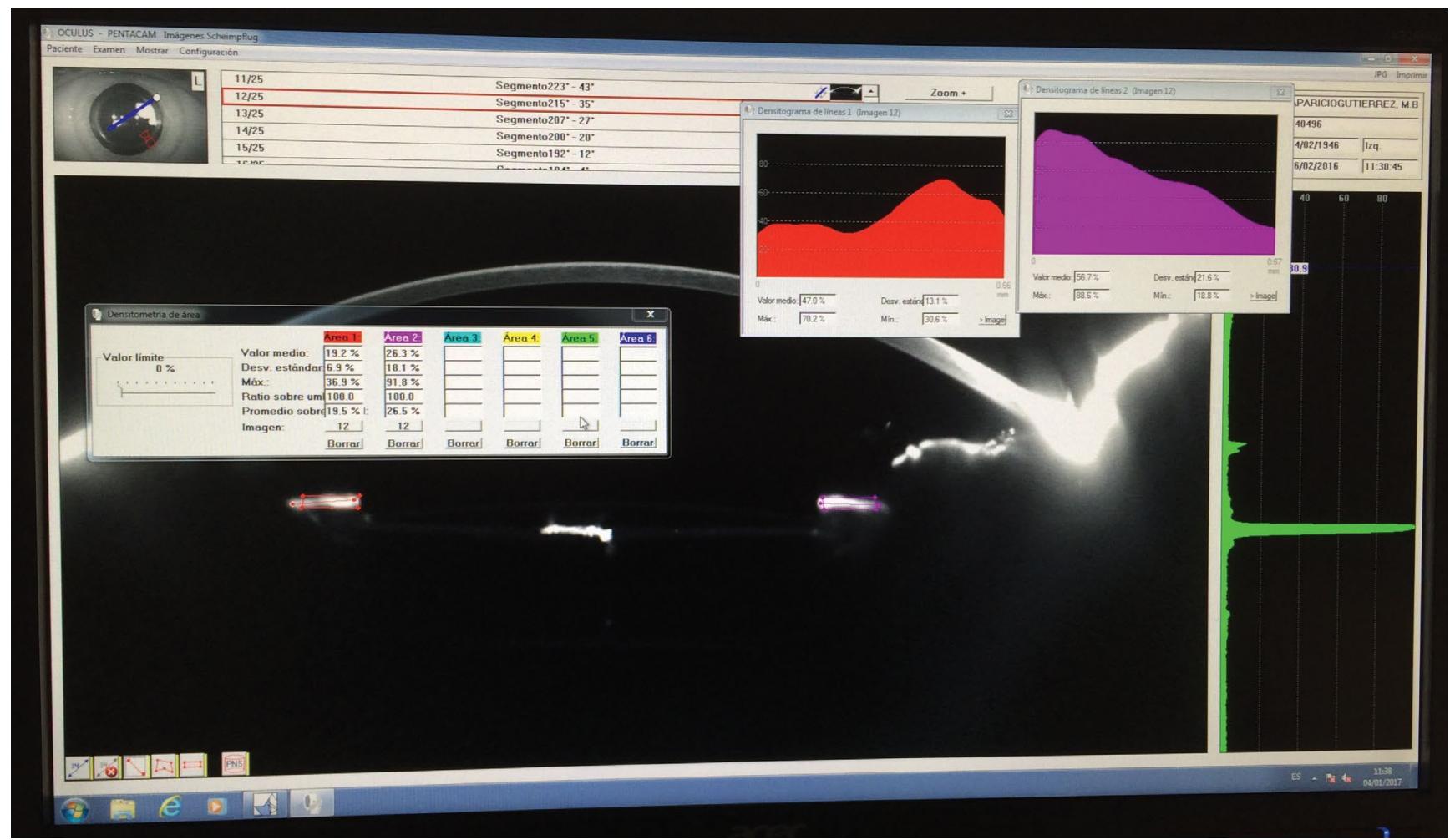

Figure 1: Using Oculus Pentacam ${ }^{\circledR} \mathrm{HR}$ densitometry software a very high Peak densitometry level appears automatically on the right side of the screen at the height of the intraocular lens while each selected meridian is analyzed. This high value represents the intraocular lens optics iatrogenically damaged.

Using Oculus Pentacam ${ }^{\circ}$ HR densitometry software a very high Peak densitometry level appears automatically on the right side of the screen at the height of the damaged intraocular lens while each selected meridian is analyzed (Figure 1).

The patient referred progressive diminution of the dysphotopsias and in the third month examinatination informed us of the resolution of these symptoms.

\section{Discussion}

The Pentacam ${ }^{\circledR}$ HR software calculates three types of lens capsule densitometry (area, linear and peak) and provides density levels as an easy, rapid, repeatable assessment. In 2016 Alberdi, et al. measuring anterior capsule opacification found that the density values provided by area and linear densitometry methods have a strong positive correlation with the levels obtained by the Slit-lamp based photographic subjective scale [4]. In the same study, they found a little or no correlation between peak densitometry and the subjective photographic scale based on the slit lamp [4]. According to this study, peak densitometry is not an appropriate method for clinical evaluation of anterior capsule opacification [4].

Area and Linear densitometry methods are increasingly used methods to measure density of any structure of the anterior chamber.

While each selected meridian is analyzed using Scheimpflug imaging the peak densitometry level ap- pears automatically on the right side of the screen. The peak densitometry value is the point at which the lens densitometry is highest on the densitogram. Therefore, it is not useful to measure the average density of structures of the anterior chamber. For this reason, peak densitometry has other clinical applications, for example for finding intralenticular foreign bodies $[5,6]$ (Figure 2).

In this article, we propose the density levels provided by Oculus Pentacam ${ }^{\circledR} \mathrm{HR}$ densitometry Peak method as a diagnostic tool to evaluate lens damage after cataract surgery.

The acquisition of Scheimpflug images must be performed according to standard procedures to avoid the appearance of artifacts and to perform an error-free analysis. Densitometry measurements of anterior chamber structures may be impossible or distorted by external factors such as insufficient mydriasis or corneal opacities. The device provides a quality specification score that takes into account the area of exploration, alignment and eye movements. This quality specification score helps users evaluate the validity of each image acquisition [1].

\section{Ethical Statement}

The authors of this paper have no financial or proprietary interest in any of the products mentioned in this article nor have they received financial support from any of the following multinational companies:

- Oculus (Pentacam ${ }^{\circledR} \mathrm{HR}$ ) Wetzlar-Germany 


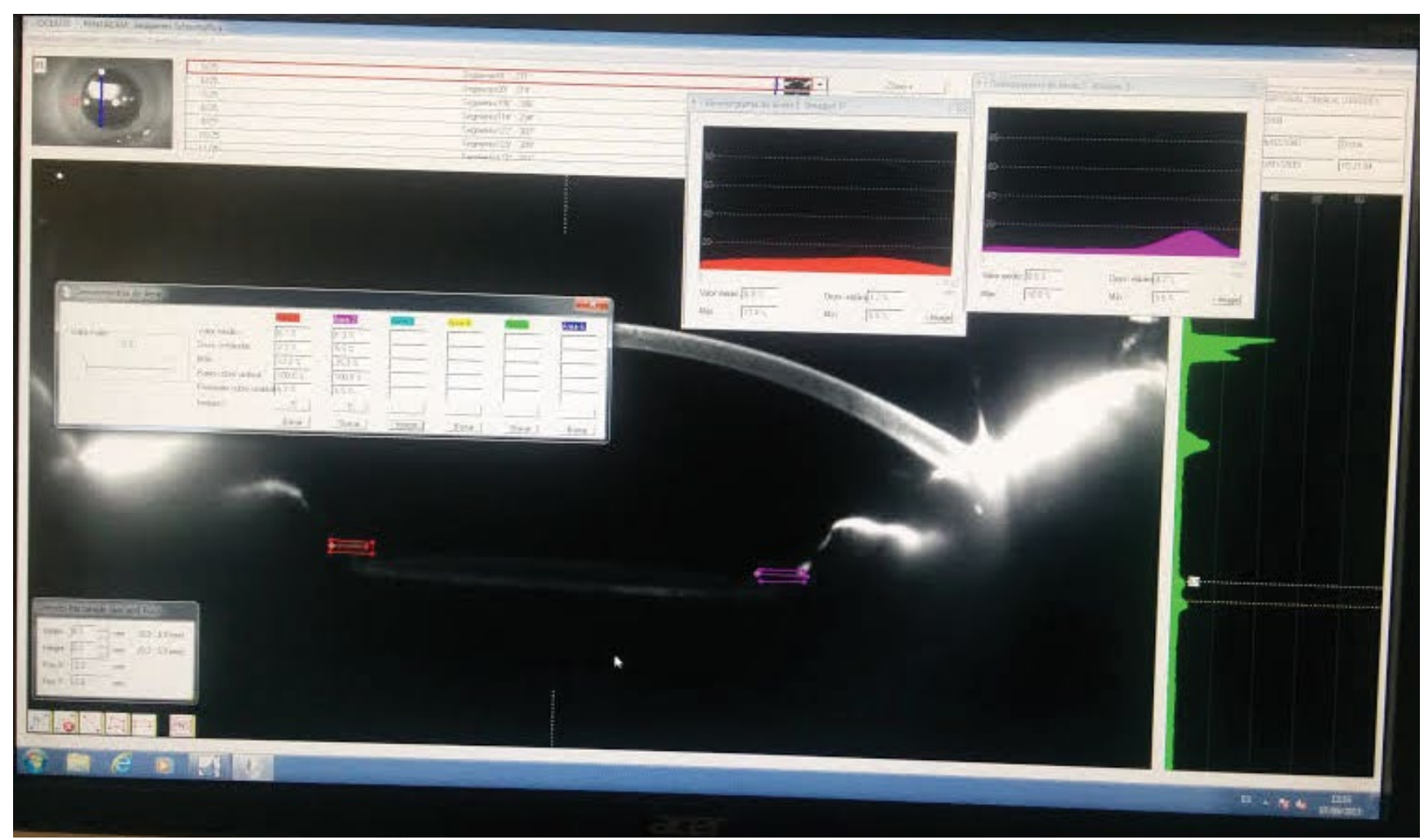

Figure 2: In this other figure we can see an intraocular lens without damage in its optic so a small elevation corresponding to the anterior edge of the intraocular lens and a small elevation that corresponds to the posterior edge of the optic appears on the right side of the screen (Peak densitometry values). In this case the opacification of the anterior capsule does not generate elevation in this zone.

\section{References}

1. Rabsilber TM, Khoramnia R, Auffarth GU (2006) Anterior chamber measurements using Pentacam rotating Scheimpflug camera. J Cataract Refract Surg 32: 456-459.

2. Kirkwood BJ, Hendicott PL, Read SA, Pesudovs K (2009) Repeatability and validity of lens densitometry measured with Scheimpflug imaging. J Cataract Refract Surg 35: 1210-1215.

3. S Harsum, S Mann, I Clatworthy, J Lewin, B Little (2010) An investigation of intraocular lens damage and foreign bodies using an injectable hydrophilic acrylic lens implant. Eye 24: 152-157.
4. Alberdi T, Mendicute J, Bascarán L, Goñi N, Barandika O, et al. (2016) Anterior capsule opacification after femtosecond laser-assisted cataract surgery: Clinical classification versus Scheimpflug device densitometry values. J Cataract Refract Surg 42: 826-832.

5. Singh R, Ram J, Gupta R (2015) Use of Scheimpflug imaging in the management of intra-lenticular foreign body. Nepal J Ophthalmol 7: 82-84.

6. Grewal SPS, Jain R, Gupta R, Grewal D (2006) Role of Scheimpflug Imaging in Traumatic Intralenticular Foreign Body. Am J Ophthalmol 142: 675-676. 